Pirineos, 164: 33 a 48, JACA; 2009. ISSN 0373-2568

\title{
CLIMA E INCENDIOS FORESTALES EN CANTABRIA: EVOLUCIÓN Y TENDENCIAS RECIENTES
}

\author{
V. Carracedo Martín, C. Diego liaño, J. C. García Codrón \& D. F. Rasilla Álvarez \\ Departamento de Geografía, Urbanismo y Ordenación del Territorio. Universidad de Cantabria \\ Avda. Los Castros, s/n. 39005 Santander \\ c-e: virginia.carracedo@unican.es
}

\begin{abstract}
Wildfires are a frequent event in Cantabria, but their seasonality does not match the typical warm season maximum generalized in most of the Iberian Peninsula. They occur at the end of the winter and the beginning of the spring (January to March), being mostly anthropogenically triggered, in conjunction with "Suradas", a windstorm which combines high winds and low humidity. In this contribution temporal trends of several climatic variables from meteorological observatories located in Cantabria and nearby regions are analyzed since 1961 during the highest risk period in order to assess to what extent the occurrence of wildfires may be linked to the recent climatic variability. Our results show that the regional climate has become warmer and drier, due to the combined effects of increases in temperatures and the decrease in relative humidity and precipitation, variables that are likely to play an important role in drought and fire proneness. However, the exception is the frequency of "Suradas", which have reduced. Those regional climatic trends are strongly linked to the recent evolution of atmospheric circulation at regional and hemispheric scale. The higher frequency of anticyclonic cells over the Iberian Peninsula, and conversely, the reduction of the number of Atlantic baroclinic disturbances are consistent with the temporal evolution of the North Atlantic Oscillation.
\end{abstract}

Keywords: wildfires, Cantabria, climate variability, windstorms.

RESUMEN.- Los incendios son un fenómeno frecuente en Cantabria, pero su estacionalidad no se corresponde con el típico máximo estival característico del resto de España. La mayoría se concentran a fines del invierno y comienzos de la primavera y su origen son unas prácticas ganaderas (principalmente la quema de matorral para mantenimiento de pastos) que aprovechan las favorables condiciones atmosféricas originadas por un tipo de temporal de viento conocido localmente como "surada". En 
este artículo se analizan las tendencias de algunas variables climáticas, desde 1961, durante el periodo de máximo riesgo de incendios para determinar en qué medida la aparición de incendios forestales ha estado condicionada por la evolución climática reciente. Los resultados muestran que el clima regional ha experimentado un calentamiento y un desecamiento, debido al efecto combinado del incremento de la temperatura y de la reducción de humedad relativa y precipitación, variables que juegan un papel determinante en la aparición de este fenómeno. Sin embargo, la frecuencia de "suradas" ha disminuido. Esta evolución climática regional está estrechamente vinculada con la evolución de la circulación atmosférica a escala sinóptica y hemisférica; la mayor frecuencia de células anticiclónicas sobre la Península Ibérica a fines del invierno y comienzo de la primavera, y la reducción del número de perturbaciones atlánticas concuerdan en gran medida con la evolución de la Oscilación del Atlántico Norte.

Palabras clave: incendios forestales, Cantabria, variabilidad climática, temporales de viento.

\section{Introducción}

A pesar de su clima húmedo y lluvioso, los incendios forestales constituyen, paradójicamente, un fenómeno frecuente en Cantabria. De hecho, desde 1968, el primer año con datos de incendios para la región, se han producido en torno a 11.500 fuegos que han quemado una superficie de más de 160.000 ha, lo que equivale a un $45 \%$ de la superficie forestal regional. Estos incendios han afectado principalmente a superficies no arboladas -éstas suponen más del $85 \%$ - y son responsables de cuantiosos daños, no sólo medioambientales sino también económicos.

Otro aspecto singular es su distribución mensual (Figura 1), ajena a los ciclos bioclimáticos naturales, con máximos durante el cuatrimestre eneroabril, uno de los más lluviosos del año. Esta distribución estacional es resultado de la intervención humana ya que el fuego constituye una herramienta básica en las prácticas agrícolas tradicionales, habiendo sido usada desde el comienzo del asentamiento humano en el territorio, como prueban numerosas evidencias arqueológicas (CADENAS, 1999).

El número de incendios registrados en Cantabria desde 1968 muestra una aparente tendencia ascendente, aunque su fiabilidad debe matizarse, ya que hasta 1990 la base de datos de incendios para España no recogía todos los incendios, generalmente los más pequeños; si analizamos la evolución de este parámetro a partir de dicho año no se detecta una tendencia significativa. La ausencia de una evolución clara a largo plazo se observa igualmente en el caso de la superficie quemada. No obstante, se detectan variaciones inter- 

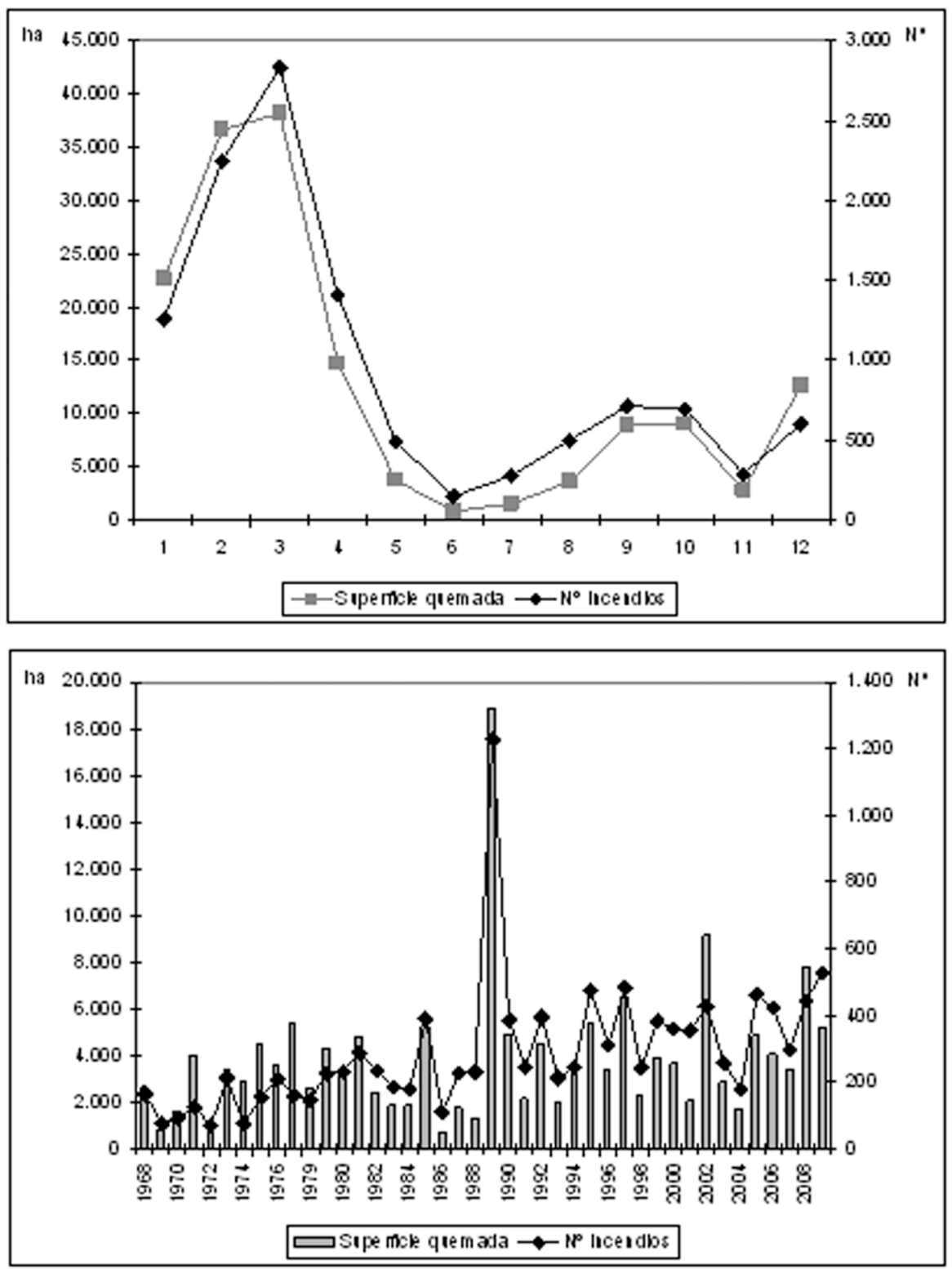

Figura 1. Total mensual (arriba) y variabilidad interanual (abajo) del número de incendios y superficie quemada (ha) en Cantabria (1968-sep 2009).

Figure 1. Monthly total (above) and interanual variability (below) of the number of fires and burned surface (ha) in Cantabria (1968-2009). 
anuales significativas, vinculadas a episodios de gran actividad, como los años 1989, 2002 y 2008, que están relacionados con factores climáticos (CARRACEDO et al., 2006). En este sentido, los fuegos en el litoral cantábrico no se alejan de los resultados de estudios similares llevados a cabo en otras regiones del globo, que señalan cómo la velocidad de propagación de los fuegos, y por lo tanto, las superficies quemadas, dependen estrechamente de las condiciones ambientales (PYNE et al., 1996; WESTERLING et al., 2004; PEREIRA et al., 2005). Los vínculos entre la evolución reciente del clima a escala regional y los incendios forestales han sido analizados en los Alpes (REINHARD et al., 2005), el SW de EEUU (CRIMMINS \& COMRIE, 2004) y el mediterráneo español (PIÑOL et al., 1998; PAUSAS, 2004).

Siguiendo estas premisas, este trabajo describe las condiciones atmosféricas más favorables para la propagación de incendios forestales en Cantabria, y analiza su evolución en los últimos decenios. Sus resultados deberían servir para establecer futuras estrategias de gestión de los montes cantábricos ante los escenarios previstos de cambio climático (BRUNET et al., 2009).

\section{Fuentes y métodos}

Los datos de incendios utilizados proceden de la base de datos de incendios forestales de España (EGIFW) que elabora el Área de Defensa contra Incendios Forestales, dependiente del Ministerio de Medio Ambiente y Medio Rural y Marino, la cual recoge los fuegos ocurridos en Cantabria desde 1968.

La caracterización de las condiciones de la atmósfera durante los episodios de incendios en Cantabria fue emprendida combinando diversas fuentes. Con los datos diarios de temperatura, humedad relativa, dirección y velocidad del viento de los observatorios meteorológicos de Parayas (Cantabria) y Sondica (Vizcaya) se calculó un índice de riesgo de incendios forestales (IRIF) que permitió evaluar el riesgo de propagación derivado de las condiciones atmosféricas, siguiendo las directrices de la Agencia Española de Meteorología (AEMet). Este índice, derivado de su homólogo NFDRS (National FIRE Danger Rate System), operativo en EEUU hasta hace pocos años, se ajusta a los mecanismos atmosféricos que propagan los incendios forestales en Cantabria, por su capacidad para cuantificar los cambios bruscos en la capacidad potencial de desecación que tiene la atmósfera. Está basado en el cálculo de la probabilidad máxima de ignición de un fuego, usando observaciones a mediodía de temperatura y humedad relativa. Dicha proba- 
bilidad de ignición significa desde un punto de vista físico la probabilidad de que al alcanzar una fuente de ignición el combustible vegetal fino, con la humedad adaptada a las condiciones de temperatura y humedad relativa previstas, se origine un foco de incendio. El valor final, que clasifica el riesgo de incendios en 4 niveles de peligrosidad (bajo, medio, alto y extremo), se obtiene combinando las probabilidad de ignición con la velocidad y dirección del viento prevista, a partir de unos valores tabulados en los que se incorpora como variable adicional el carácter "especialmente desecante" del viento terral (INM, 1996; CADENAS et al., 1999).

El análisis de la circulación atmosférica a escala regional siguió un método objetivo de clasificación basado en índices (JENKINSON \& COLLISON, 1977). A partir de valores de presión a nivel del mar, dispuestos en una rejilla regular de $5^{\circ}$ de latitud por $10^{\circ}$ de longitud, este método se fundamenta en el cálculo de una serie de parámetros o índices cuantitativos (dirección y velocidad del flujo geostrófico y vorticidad). Cada uno de los tipos sinópticos corresponde a unos valores específicos de dichos índices, que permiten clasificar cada día en diferentes categorías. Sin embargo, los tipos de circulación obtenidos a partir de las reglas de clasificación originales no son más que configuraciones artificiales, definidos por límites arbitrarios y sin relación a priori con las condiciones en superficie. Esto puede constituir un serio impedimento que dificulte la vinculación entre la dinámica atmosférica general y la evolución de los principales parámetros climáticos sobre un ámbito espacial concreto. En consecuencia, y con el fin de ajustar dicha clasificación a las particularidades del clima de la Península Ibérica, el procedimiento original fue mejorado siguiendo dos líneas de trabajo. Por un lado, modificando el compás de la dirección del flujo y eliminando 2 categorías con escasa repercusión, lo cual redunda en una mejor interpretabilidad de los tipos advectivos; por otro lado, con el fin de superar alguna de sus limitaciones (MARTíN-VIDE, 2001), como son la abundancia de situaciones de flujo indefinido o de jornadas clasificadas como ciclónicas, se reclasificaron como anticiclónicos los días englobados en la categoría de indeterminados pero con una presión superior a $1020 \mathrm{hPa}$, mientras los ciclónicos con una presión superior a $1010 \mathrm{hPa}$ fueron a su vez incorporados al tipo indeterminado (FERNÁNDEZ GARCÍA et al., 2002). La fuente original de los valores de presión a nivel del mar fue la base de datos REANALYSIS NCEP (KALNAY et al., 1996). Finalmente, las series temporales del Índice de Severidad de Sequía de Palmer (Palmer Drought Severity Index; PDSI) fueron descargados de la página web de la Climate Research Unit (http://www.cru.uea.ac.uk/cru/projects/emulate/; VAN DER SCHRIER et al., 2006). 


\section{Resultados}

\subsection{La influencia de las condiciones atmosféricas en los incendios forestales}

Procesos atmosféricos distintos pero complementarios, actuando a diferentes escalas temporales, controlan la expansión de los fuegos en Cantabria: una variabilidad diaria, ligada a circulaciones sinópticas específicas, que disparan la actividad de los fuegos, y una variabilidad estacional, en forma de anomalías climáticas vinculadas a las oscilaciones de la circulación atmosférica a escala hemisférica, que generan unas condiciones ambientales a largo plazo favorables a los incendios.

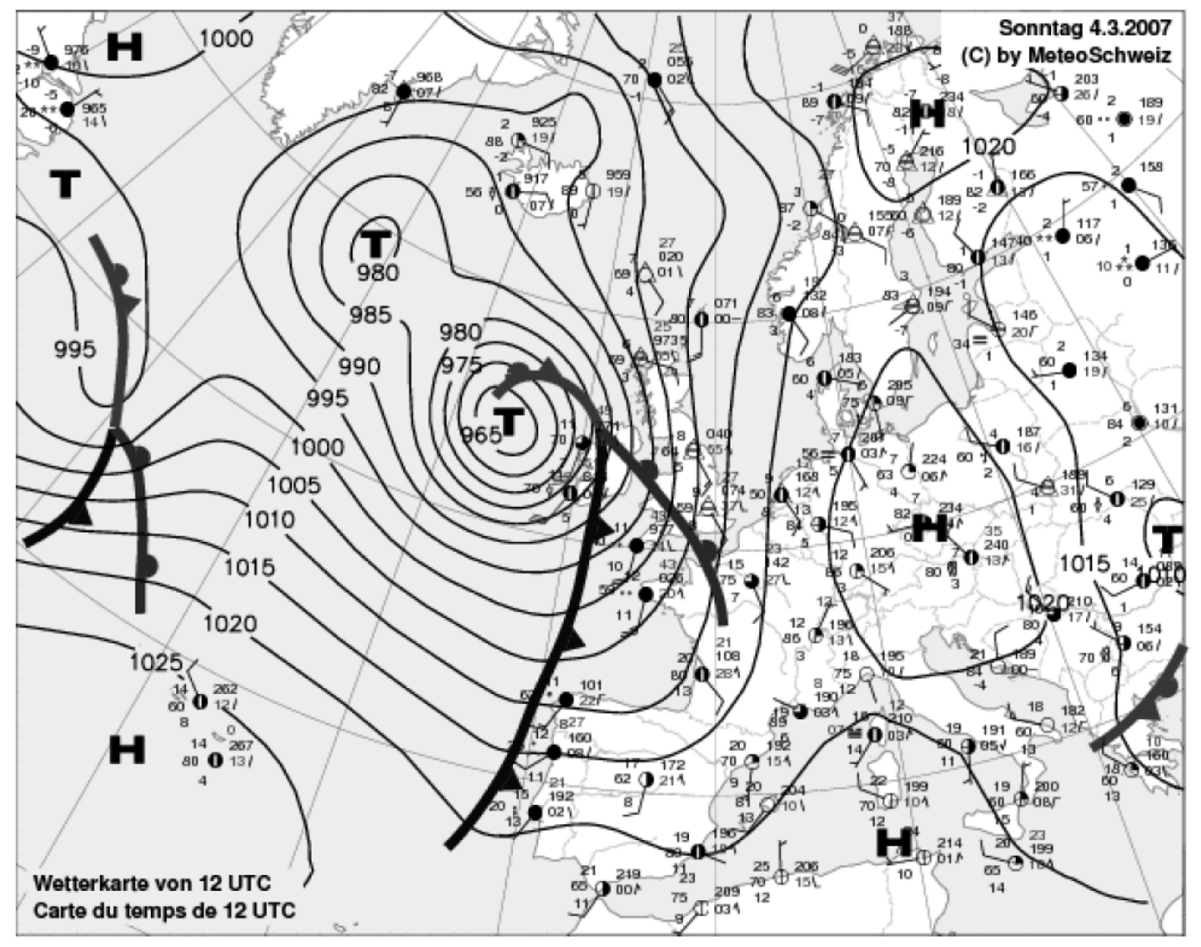

Figura 2. Situación típica correspondiente a una "surada" en Cantabria (Fuente: http:/ / www.meteossuise.com).

Figure 2. Synoptic pattern corresponding to a "surada" in Cantabria (Source: http://www.meteossuise.com). 
Es una creencia popular en Cantabria que las primeras derivan de unos temporales de viento denominados "surada"; algunos hechos históricos apoyan esta creencia, como el fuego que destruyó gran parte del centro de la ciudad de Santander en 1941. Las "suradas" constituyen un tipo de tiempo muy característico de la cornisa cantábrica, vinculado al conocido efecto "föhn" (Figura 2; RASILLA, 1999). Como consecuencia de esta situación, el contraste habitual entre ambas vertientes de la Cordillera Cantábrica se invierte, siendo la vertiente septentrional la que sufre un brusco aumento de las temperaturas (que pueden alcanzar $20^{\circ} \mathrm{C}$ en invierno, y hasta $35^{\circ} \mathrm{C}$ en otoño) y una drástica reducción de la humedad (a veces inferior al $40 \%$ ). Al calor y la sequedad le acompañan vientos normalmente fuertes (con velocidades sostenidas de $60-80 \mathrm{~km} / \mathrm{h}$ y puntas de hasta $140 \mathrm{~km} / \mathrm{h}$ ), en los que se conjugan factores sinópticos, como un abrupto gradiente de presión, y mesoescalares, derivados de la acción del relieve sobre las capas bajas de la atmósfera. Debido a estas condiciones, las "suradas" constituyen, lógicamente, una situación favorable para la propagación de los fuegos. Los tipos de circulación del SW, y en menor medida, los del SSE y W, son los más propensos a la génesis de una "surada", y como consecuencia, a la aparición de situaciones de elevado riesgo de incendios en Cantabria (Figura 3). El resto de tipos de circulación (anticiclónico -A-, del Este -E-, del NNW y del NNE) contribuye de manera anecdótica a la génesis de niveles elevados de riesgo.

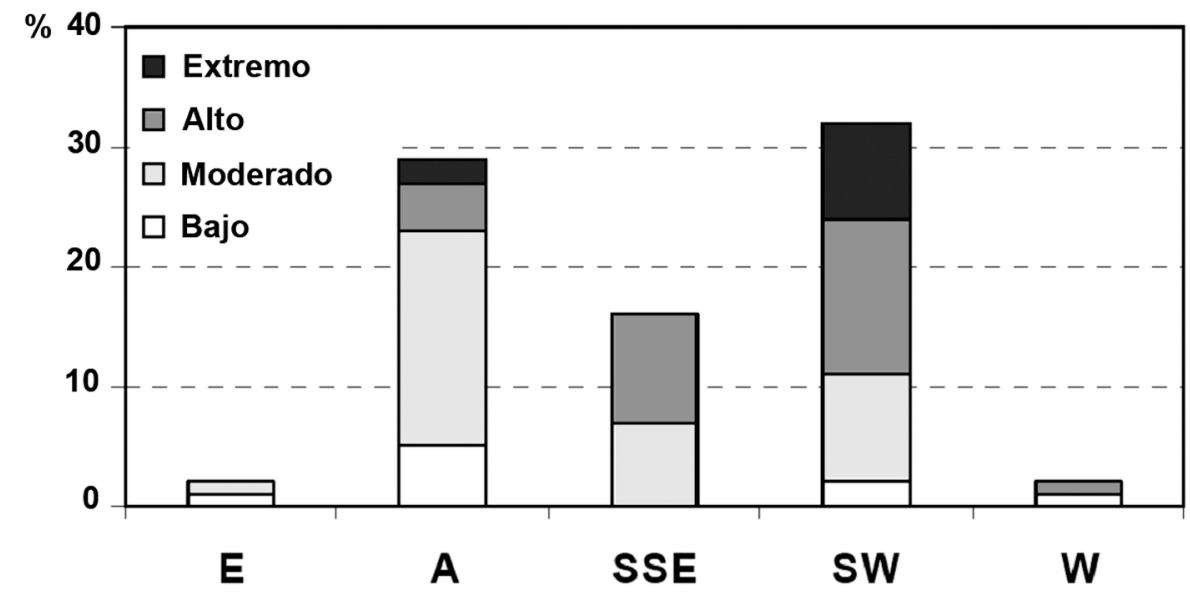

Figura 3. Frecuencia de condiciones atmosférica según el riesgo de incendio en Cantabria según tipo de circulación, correspondiente al $5 \%$ de los días de mayor superficie quemada en Cantabria.

Figure 3. Frequency of atmospheric conditions prone to fire risk in Cantabria according to the circulation type corresponding to the $5 \%$ of the days with the higher surface burned in Cantabria. 
Para verificar la hipótesis de una relación entre incendios, valores elevados de riesgo y "suradas" se examinó también la correspondencia entre tipos de circulación y niveles de riesgo de incendios durante los días con mayor número de incendios y más superficie quemada. Dado que la mayor parte de los grandes episodios de incendios se concentran en un número reducido de días, se seleccionaron las jornadas en las que la superficie quemada superaba las 90 ha, umbral que equivale al percentil 95 de la superficie diaria quemada en Cantabria.

La existencia de condiciones de alto riesgo no siempre asegura la génesis de este tipo de episodios; por otro lado, no es infrecuente que algunos días que registran valores elevados de superficie quemada ocurran bajo condiciones atmosféricas relativamente inapropiadas. En estos casos, esta discrepancia se explica por la intercalación, dentro de episodios favorables, de días con menor riesgo, lo que ocurre, por ejemplo, cuando los extremos de las discontinuidades frontales asociadas a las borrascas atlánticas cruzan la región. Estas condiciones interrumpen el calor y la sequedad, propiciando un giro de los vientos hacia el $\mathrm{W}$ y NW, acompañados de precipitaciones ligeras. Sin embargo, los fuegos persisten debido a la necesidad de generar pastos en los momentos previos a la subida del ganado a lo largo de la primavera (CARRACEDO et al., 2007).

A ello debe añadirse el papel de otros factores, como es la aparición de condiciones de sequía severa. Para contrastar ambos fenómenos, se ha comparado el número de incendios forestales y la superficie quemada mensual con los valores simultáneos del Índice de Sequía de Palmer (Figura 4), usado

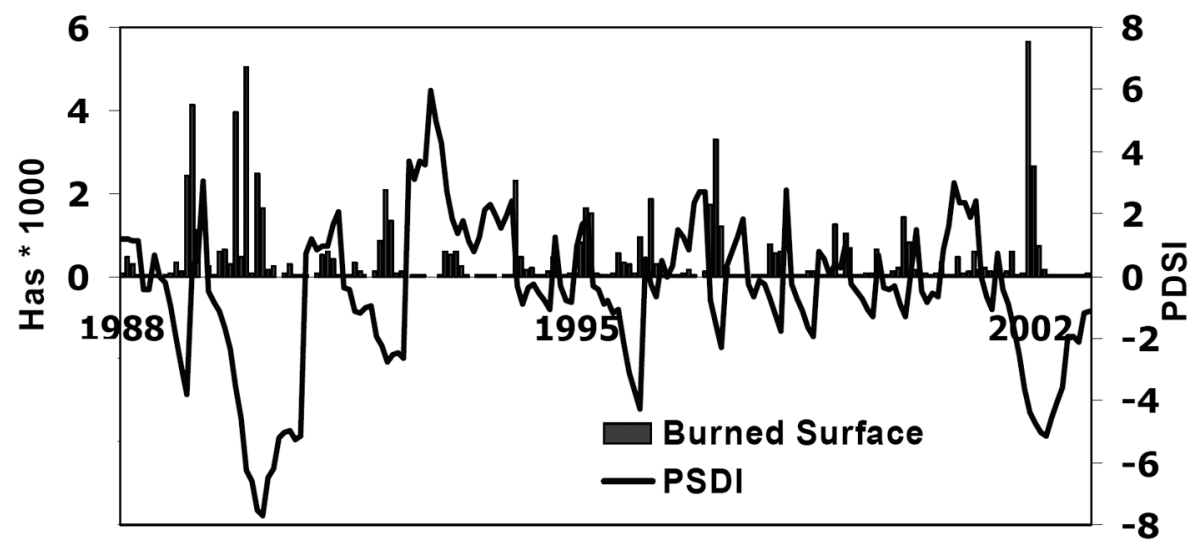

Figura 4. Evolución mensual de la superficie quemada y del índice PDSI en Cantabria (1988-2002). Figure 4. Monthly evolution of the surface burnt and the PDSI in Cantabria (1988-2002). 
frecuentemente para caracterizar las condiciones de sequía a escala global y su relación con los incendios forestales (KEELEY, 2004). El episodio de sequía más relevante en Cantabria tuvo lugar entre 1988 y 1990, alcanzando su momento álgido durante el trimestre enero-marzo de 1990 (Figura 5). La persistencia de altas presiones en el Mediterráneo Occidental condujo a un predominio de situaciones del SW sobre la Península Ibérica. Este tipo de configuración es propia de episodios intensos de sequía en el área cantábrica, Alto Ebro y suroeste de Francia, mientras en el resto de la Península Ibérica las condiciones suelen ser más benignas, ya que ese mismo tipo de circulación produce habitualmente la llegada de masas húmedas desde el Atlántico. Otro episodio de menor severidad se produjo a lo largo del año 2002, mostrando similitudes con la aquí mostrada.
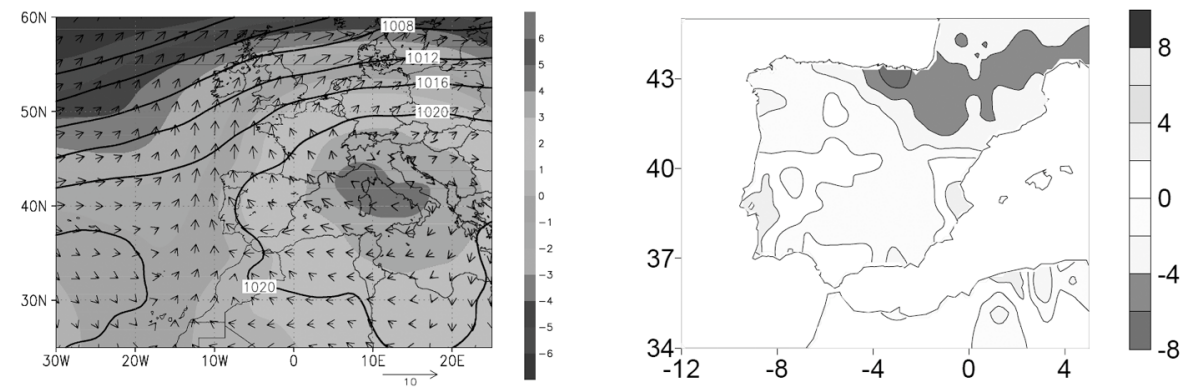

Figura 5. Anomalías de la presión a nivel del mar y del viento entre noviembre y marzo de 1990 y patrón espacial del índice PDSI en la Península Ibérica correspondiente a marzo de 1990. Figure 5. Sea Level Pressure and wind anomalies between November and March 1990 and the PDSI pattern corresponding to March 1990.

\subsection{Tendencias climáticas recientes y susceptibilidad a los incendios}

Una vez se han definido los factores atmosféricos favorables a la aparición de grandes episodios de incendios, el siguiente paso es analizar cómo han progresado dichos factores en las últimas décadas.

Con el fin de analizar la evolución temporal de los mecanismos atmosféricos a corto plazo se ha comparado la variabilidad interanual de las "suradas" con la frecuencia de días clasificados según el Índice de Riesgo de Incendios en el trimestre enero-marzo desde 1961 hasta la actualidad. El aspecto más relevante es una disminución del número y duración de las "suradas", que viene a coincidir con una disminución de la frecuencia de los 
días clasificados dentro de los tipos sinópticos del SSW y SSE, citados en líneas anteriores como los más favorables al desencadenamiento de este fenómeno y de días de alto riesgo de incendios (Figura 6).
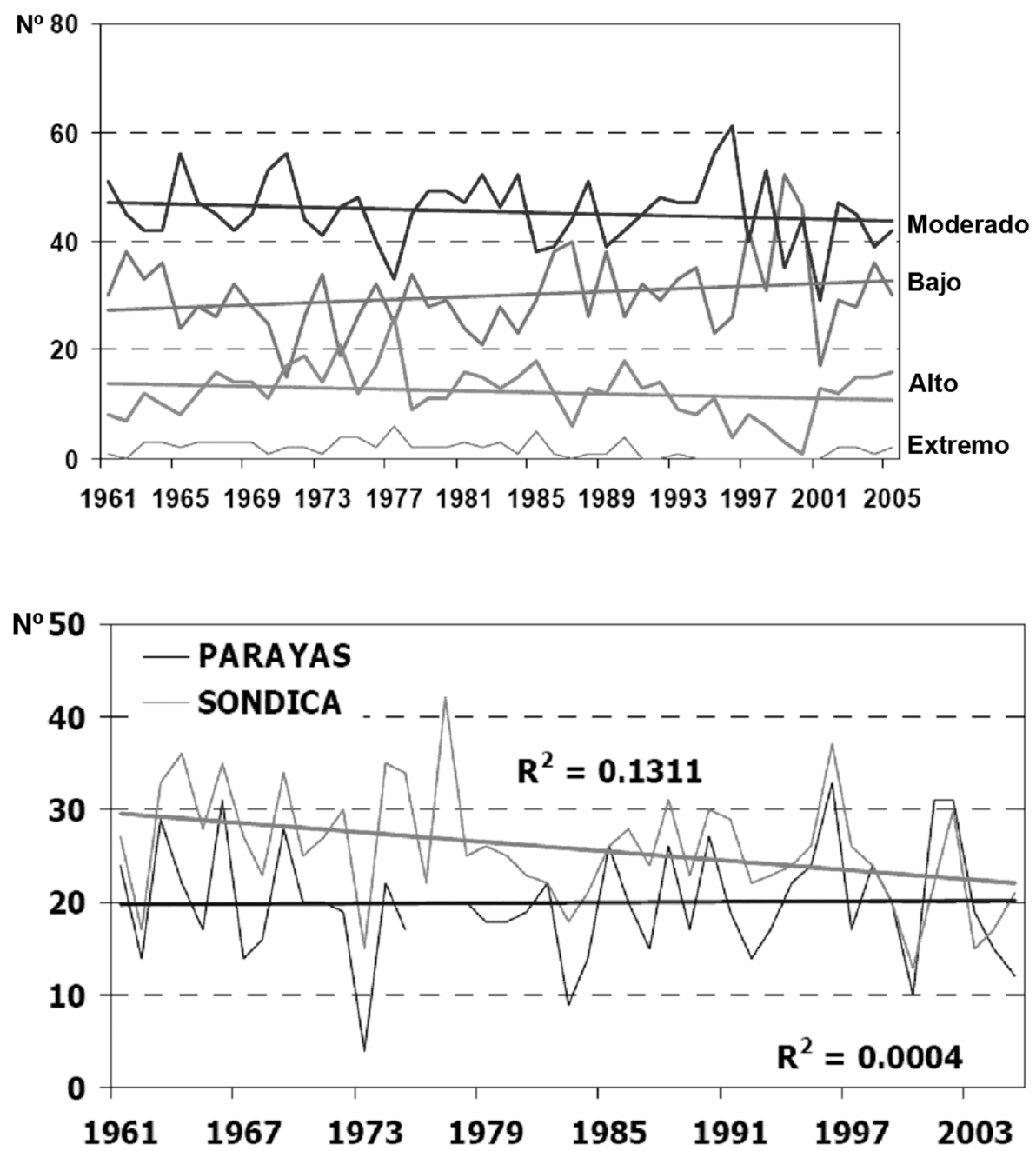

Figura 6. Evolución temporal de los días según categoría de riesgo de incendios (arriba) y "Suradas" (abajo) en Cantabria.

Figure 6. Temporal evolution of the days according the categories of forest fire risk (above) and "Suradas" (below) in Cantabria. 
Esta tendencia a la disminución de las condiciones de riesgo de incendios en Cantabria contrasta, sin embargo, con una mayor frecuencia de episodios de sequía en la región, según se desprende de la evolución del índice PDSI (Figura 7). Con anterioridad a 1979, los valores negativos del PDSI, que señalan condiciones de sequía, muestran una frecuencia menor y una baja intensidad; sin embargo, durante las décadas de los 80 y 90 aumentó considerablemente la variabilidad de la serie, apareciendo un mayor número de episodios con valores negativos, entre los cuales destacaron por su intensidad y duración los anteriormente mencionados de 1988-1990 y 2002. Aunque las conclusiones son preliminares, la prevalencia de estos episodios podría atribuirse a una disminución de la cuantía de las precipitaciones (sobre todo, de los días de precipitación intensa), no a una disminución de su frecuencia, y al aumento generalizado de las temperaturas (Figura 8).

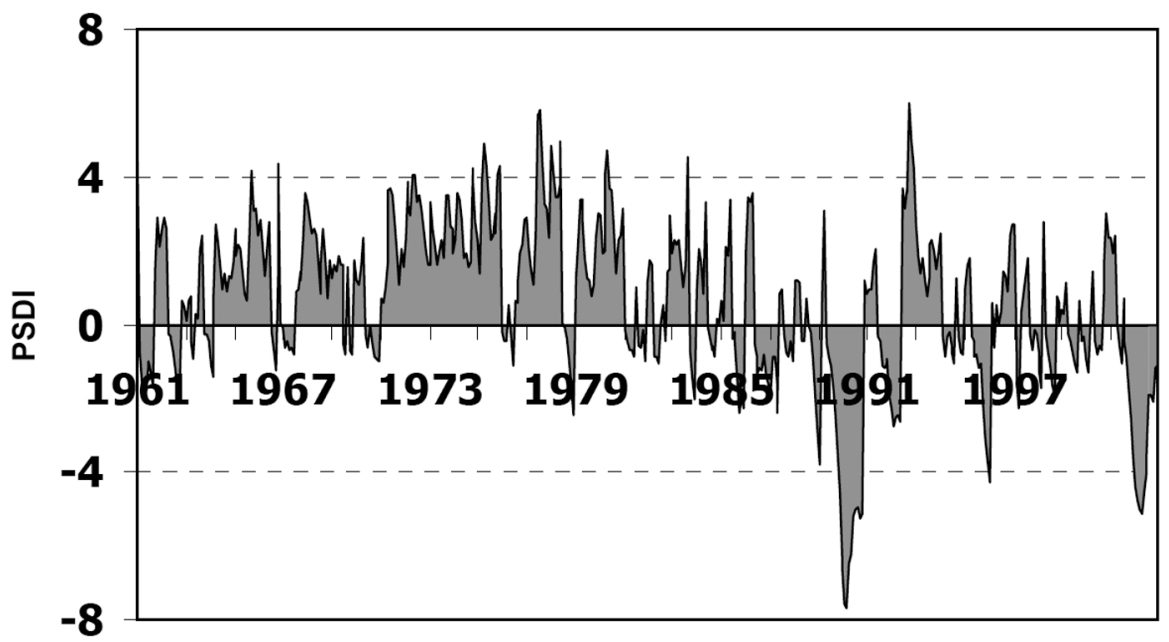

Figura 7. Evolución del Índice de Severidad de Sequía de Palmer (PDSI) entre 1961-2002 en Cantabria.

Figure 7. Evolution of the PDSI in Cantabria (1961-2002).

La evolución climática local de este sector de la Península Ibérica está estrechamente vinculada a la evolución de la circulación atmosférica a escala regional. A partir de las series diarias de tipos de circulación, parece evidente que el descenso en las "suradas" se corresponde con una reducción de las situaciones sinópticas propicias a este fenómeno (fundamentalmente SSW y 
SSE), compensadas por un aumento de las situaciones de "buen tiempo" (anticiclónico, E y NNE), que corresponden a los días caracterizados por situaciones de bajo riesgo (Figura 9). Y al mismo tiempo, esa evolución regional responde a las fluctuaciones de la circulación atmosférica a escala hemisférica y frecuencia interanual, representada por dos teleconexiones, la Oscilación del Atlántico Norte y la Oscilación del Mediterráneo Occidental (Tabla 1). En el caso de la NAO, la sustitución de la fase negativa de las décadas de los 60 y 70 por una fase positiva en los 80 y 90 ha supuesto no sólo la reducción del número de circulaciones del SSW, las principales causantes de las "suradas" y de los días de alto riesgo de incendios, sino también una disminución de la precipitación invernal y un aumento de las temperaturas, que podría sustentar la hipótesis de una tendencia hacia condiciones menos húmedas en el Cantábrico.

\section{Conclusiones}

Los incendios forestales son un hecho habitual en el mundo rural cántabro, pero su estacionalidad no se ajusta al modelo mediterráneo imperante en la mayor parte de la Península Ibérica. En Cantabria la mayoría de estos sucesos ocurre a finales del invierno y comienzo de la primavera beneficiándose, en su mayoría, de situaciones atmosféricas concretas, como las "suradas", y de condiciones de sequía. El peso de los factores atmosféricos a corto y medio plazo está siendo modificado en las últimas décadas a causa de la evolución climática de la región. La disminución en el número de "suradas" reduce la frecuencia de días de alto riesgo de incendios, a causa de la disminución de las perturbaciones atlánticas, lo cual se corresponde con una fase positiva de la Oscilación del Atlántico Norte. A su vez, el calentamiento y desecamiento de la región, por la mayor frecuencia e intensidad de las situaciones de estabilidad atmosférica, ha generado en las dos últimas décadas episodios de sequía desconocidos en los años precedentes, contrarrestando la disminución del riesgo de incendios a corto plazo.

No obstante, dada la fuerte dependencia del fenómeno de los incendios respecto de la actividad ganadera en esta región, el diseño de estrategias futuras de prevención y la realización de escenarios de incendios que tengan en cuenta la evolución prevista del sistema climático, obligan a incorporar el componente humano en ellos. 

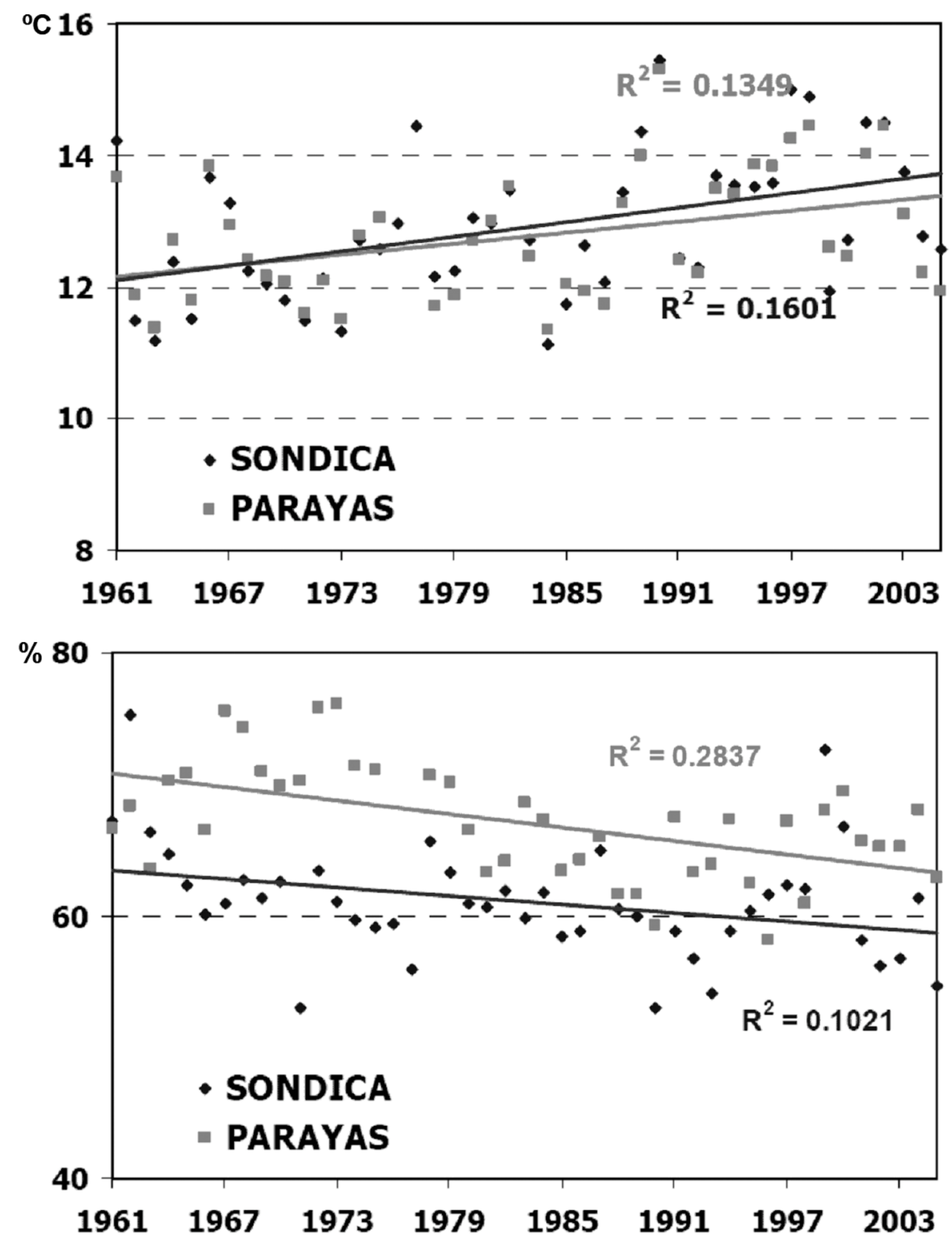

Figura 8. Tendencias en las temperaturas máximas diarias y la humedad relativa a las 12 UTC en Cantabria desde 1961 (Periodo enero a marzo).

Figure 8. Trends in daily maximum temperatura and relative humidity at 12 UTC in Cantabria from 1961 (January to March). 


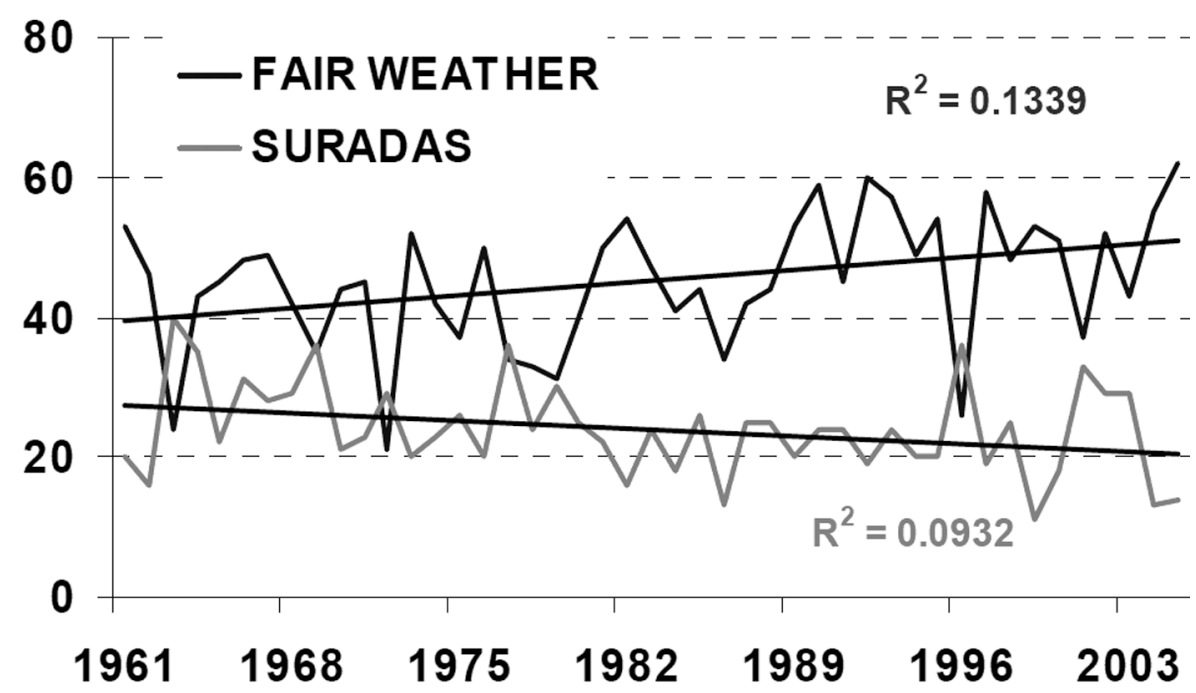

Figura 9. Frecuencia (días) de tipos de circulación seleccionados entre 1961 y 2005 (periodo enero a marzo).

Figure 9. Frequency (days) of weather types selected between 1961 and 2005 (January to March).

Tabla 1. Correlación (coeficiente " $r$ " de Pearson) entre el índice NAO y la frecuencia y parámetros climáticos por tipo de condiciones atmosféricas ( ${ }^{* *}$ significativo al $99 \%$; ${ }^{*}$ significativo al $95 \%$ ). Table 1. R-Pearson correlation between the NAO index and the frequency of weather types and some climate variables (** significant at $99 \%,{ }^{*}$ significant at $95 \%$ ).

\begin{tabular}{|lcccc|}
\hline & Observatorio & Frecuencia & Temperatura & Humedad relativa \\
\hline Situaciones de & Parayas & $0,59^{* *}$ & $0,51^{* *}$ & $-0,23^{*}$ \\
“buen tiempo" & Sondica & & $0,44^{* *}$ & $-0,23^{*}$ \\
\hline Suradas & Parayas & $-0,56^{* *}$ & 0,11 & $-0,18$ \\
& Sondica & & $0,21^{*}$ & $-0,37^{\star *}$ \\
\hline
\end{tabular}

\section{Referencias}

BRUNET, M., CASADO, M.J., DE CASTRO, M., GALÁN, P., LÓPEZ, J.A., MARTÍN, J.M., PASTOR, A., PETISCO, E., RAMOS, P., RIBALAYGUA, J., RODRÍGUEZ, E., SANZ, I. \& TORRES, L. (2009). Generación de escenarios regionalizados de cambio climático para España. Agencia Estatal de 
Meteorología, Ministerio de Medio Ambiente, Medio Rural y Marino, 165 pp.

CADENAS, I.; MESTRE, A. \& MORENO, T. (1999). Estudio sobre la bondad del ajuste de incendios forestales utilizado en la campaña nacional. Informe final del estudio. SAM. INM.

CARRACEDO, V., LIAÑO, C., GARCÍA CODRÓN, J.C. \& RASILLA ÁLVAREZ, D. (2006). An advance of the role of human and natural factors in the genesis of forest fires in Cantabria (Northern Spain). V Intl Congress on Forest Fire Research - V ICFFR. Figueira da Foz, Portugal. Forest Ecology and Management, vol 234, Sup. 1.

CRIMMINS, M.A. \& COMRIE, A.C., (2004). Wildfire-Climate Interactions across Southeast Arizona. International Journal of Wildland Fire, 13: 455-466.

FERNÁNDEZ GARCÍA, F.; RASILLA ÁLVAREZ, D. F. \& HORCAS, R. (2002). Secular variations of the synoptic scale atmospheric circulation over the Iberian Peninsula. Detecting and Modelling Regional Climate Change (Brunet India, M. y López Bonillo, eds), Springer-Verlag: 229-238.

INM, (1996) Normativa de la campaña nacional de apoyo meteorológico a la lucha contra incendios forestales.

JENKINSON, A.F. \& COLLISON, B.P. (1977). An initial climatology of gales over the North Sea. Synoptic Climatological. Branch Memory, UKMO, 62 pp.

KALNAY, E., et al., (1996). The NCEP/NCAR 40-Year Reanalysis Project. Bulletin of the American Meteorological Society, 77: 437-471.

KEELEY, J.E. (2004). Impact of antecedent climate on fire regimes in coastal California. International Journal of Wildland Fire 13: 173-182.

MARTÍN-VIDE, J. (2001) Limitations of an objective weather-typing system for the Iberian Peninsula. Weather, 56: 7-10.

PAUSAS, J.G. (2004) Changes in fire and climate in the Eastern Iberian Peninsula (Mediterranean Basin). Climatic Change, 63: 337-350.

PEREIRA, M.G., TRIGO, R.M., DA CAMARA, C.C., PEREIRA, J.M.C., LEITE, S.M., (2005). Synoptic patterns associated with large summer forest fires in Portugal. Agricultural and Forest Meteorology, 129: 11-25.

PIÑOL, J., TERRADAS, J., \& LLORET, F. (1998). Climate Warming, Wildfire Hazard, and Wildfire Occurrence in Coastal Eastern Spain. Clim. Change 38: 345-357.

PYNE, S.J., ANDREWS, P.L. \& LAVEN, R.D. (1996). Introduction to Wildland Fire. John Wiley and Sons, New York.

RASILLA ÁLVAREZ, D. F., (1999). Viento sur y efecto föhn en la Cornisa Cantábrica: impactos climáticos regionales. CEDEX, Madrid.

REINHARD, M., REBETEZ, M. \& SCHLAEPFER, R. (2005). Recent Climate Change: Rethinking Drought in the Context of Forest Fire Research in Ticino, South of Switzerland. Theoretical and Applied Climatology, 82: 17-25. 
VAN DER SCHRIER, G., BRIFFA, K.R., JONES, P.D. \& OSBORN, T.J. (2006). Summer moisture variability across Europe. Journal of Climate 19: 28182834.

WESTERLING, A.L., HIDALGO, H.G., CAYAN, D.R. \& SWETNAM, T.W. (2006). Warming and Earlier Spring Increase Western U.S. Forest Wildfire Activity. Science, 313: 940-943. 